Pacific

Journal of

Mathematics

UNIQUENESS RESULTS

FOR CONSTANT MEAN CURVATURE GRAPHS

LAURENT MAZET

Volume $230 \quad$ No. 2

April 2007 


\title{
UNIQUENESS RESULTS FOR CONSTANT MEAN CURVATURE GRAPHS
}

\author{
LAURENT MAZET
}

\begin{abstract}
We give two uniqueness results for the Dirichlet problem associated to the constant mean curvature equation, involving mean curvature graphs over strips of $R^{2}$. The proofs are based on height estimates and the study of the asymptotic behavior of solutions to the Dirichlet problem.
\end{abstract}

\section{Introduction}

Surfaces with constant mean curvature are of great interest in mathematics: they model soap films, for example, and appear as interfaces in isoperimetric problems. One viewpoint in studying such surfaces is to consider them as graphs.

Let $\Omega$ be a domain of $\mathbb{R}^{2}$. The graph of a function $u$ over $\Omega$ has constant mean curvature $H>0$ if it satisfies the partial differential equation

$$
\operatorname{div} \frac{\nabla u}{\sqrt{1+|\nabla u|^{2}}}=2 H \text {. }
$$

Thanks to the work of J. Serrin [1970; 1969] and J. Spruck [1972/73], we can build a lot of constant mean curvature graphs over bounded domains of $\mathbb{R}^{2}$. Over unbounded domains, the Dirichlet problem associated to (CMC) is more complicated. R. Finn [1965] asked whether the graph of a solution $u$ of (CMC) over the strip $\mathbb{R} \times(-1 /(2 H), 1 /(2 H))$ must be a regular cylinder of radius $1 /(2 H)$. P. Collin [1990] and A. N. Wang [1990] then built counterexamples. Other examples of solutions over strips were given by R. López [2001; 2002].

Our key results in this paper, Theorems 10 and 12, say that solutions are unique under the conditions of either the Collin-Wang or the López examples. These examples are of particular interest because they include unbounded boundary data; uniqueness is already known in the case of bounded boundary data, and also when the boundary data is small with respect to $\ln r$, where $r$ is the distance to the origin [Huang 1995].

Our proofs involve two major steps. First, if there are two solutions for the same boundary data, the difference between these solutions cannot stay bounded. This

MSC2000: 53A10.

Keywords: constant mean curvature, uniqueness in Dirichlet problem. 
yields information on the asymptotic behavior of the boundary data. In the second step, we analyze the consequences of this behavior for the asymptotic behavior of a solution, using the notion of an arc of divergence. This idea is similar to the one used by Tam [1987a], who applied it to the related uniqueness question for capillary surface problems (where the desired solution $u$ of (CMC) in $\Omega$ must satisfy

$$
\frac{\nabla u}{\sqrt{1+|\nabla u|^{2}}} \cdot v=\cos \gamma \quad \text { on } \partial \Omega,
$$

rather than a Dirichlet boundary condition; here $v$ is the outward unit normal to $\partial \Omega$ and $\gamma$ is the wetting angle). See also [Tam 1987b; Hwang 1995].

The uniqueness question has been studied for the minimal surface equation

$$
\operatorname{div} \frac{\nabla u}{\sqrt{1+|\nabla u|^{2}}}=0
$$

Nitsche [1965] proved that over a strip or angular sector $\{y>|x| \cot \alpha\}$, with $0<\alpha<\pi / 2$, the only solution of (MSE) vanishing on the boundary is $u \equiv 0$. Hence he conjectured the uniqueness of the solution to the Dirichlet problem for (MSE) in such domains. Collin [1990] gave a counterexample; thus, in view of our Theorems 10 and 12, the uniqueness problem for (MSE) on strip domains stands in contrast with the same problem for (CMC).

\section{The existence results of Collin-Wang and López}

In this section we recall two existence results on the Dirichlet problem for the constant mean curvature equation (CMC) on a strip $\Omega=\mathbb{R} \times(-l, l)$ of width $2 l$. It was proved in [López 2001] that the width needs to be at most $1 / H$ for there to be a solution. The first result we quote concerns the limiting case $2 l=1 / H$.

For $f: \mathbb{R} \rightarrow \mathbb{R}$ a continuous function, we define $\varphi_{f}$ on $\partial \Omega$ by $\varphi_{f}(x, \pm l)=f(x)$.

Theorem [Collin 1990]. Let $f: \mathbb{R} \rightarrow \mathbb{R}$ be a convex continuous function. There exists a solution $u$ of $(\mathrm{CMC})$ on $\Omega=\mathbb{R} \times(-1 /(2 H), 1 /(2 H))$ agreeing with $\varphi_{f}$ on the boundary.

(Wang [1990] proved this for the convex function $x \mapsto x^{2}$.)

The second result, by López, deals with the case where $2 l<1 / H$.

We say that a domain $U \subset \mathbb{R}^{2}$ satisfies an exterior $R$-circle condition if for each point $p \in \partial U$ there is a disk $D$ of radius $R$ such that $\bar{D} \cap \bar{U}=\{p\}$. This says a circle of radius $R$ can roll outside $U$ along $\partial U$ touching each point of $\partial U$.

A continuous function $f: \mathbb{R} \rightarrow \mathbb{R}$ is said to satisfy a lower $R$-circle condition if the domain $\left\{(x, y) \in \mathbb{R}^{2} \mid y \geq f(x)\right\}$ satisfies an exterior $R$-circle condition. Thus 
a circle of radius $R$ can roll under the graph of $f$ touching each point of the graph along its motion.

Theorem [López 2002]. Let $f: \mathbb{R} \rightarrow \mathbb{R}$ be a continuous function satisfying a lower $\rho_{t}$-circle condition, where $t \in \mathbb{R}_{+}^{*}$ and $\rho_{t}$ is the maximal radius of the nodoid neck with minimal radius $t$ (see below). There exists a solution $u$ of (CMC) on the strip $\Omega=\mathbb{R} \times\left(-h_{t}, h_{t}\right)$ agreeing with $\varphi_{f}$ on the boundary, where $h_{t}$ is the half-height of the same nodoid neck.

The authors of these theorems use Perron's technique to build their solutions as the supremum of subsolutions. The difficulty is finding good barrier functions to ensure the boundary value. Theorems 10 and 12 below state that the solutions built by these authors are unique for the boundary data $\varphi_{f}$.

The one-parameter family of nodoids. Constant mean curvature surfaces of revolution are of two types, each forming a one-parameter family. Unduloids are embedded surfaces: as the parameter changes, the unduloid family goes from the cylinder of radius $1 /(2 H)$ into a stack of tangent sphere of radius $1 / H$. By contrast, nodoids are not embedded; their interest lies in that each nodoid contains a piece that looks like a catenoidal neck with mean curvature vector pointing outward:

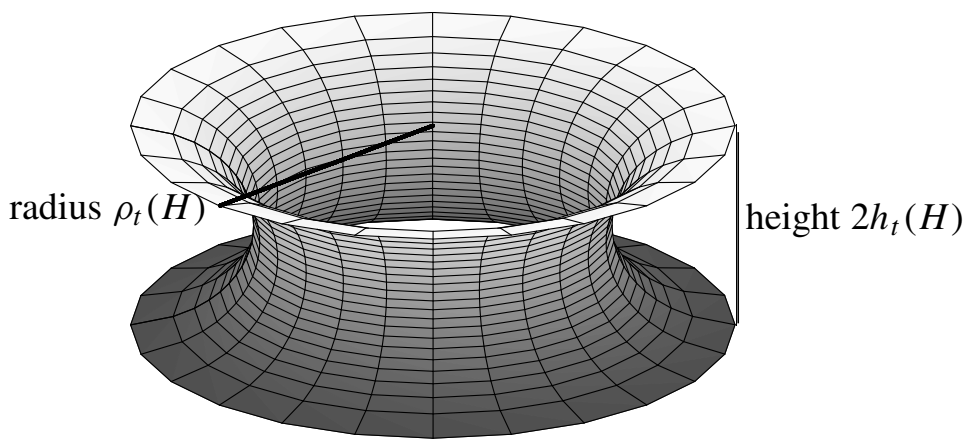

We recall the construction of nodoids and fix notations; see [Delaunay 1841; Eells 1987; López 2002] for details. Take the surface of revolution parametrized by $(r(u) \cos \theta, r(u) \sin \theta, u)$, arising from a positive smooth function $r(u)$ defined on an open interval $I$. The normal vector is

$$
N(u, \theta)=\frac{1}{\sqrt{1+r^{\prime 2}}}\left(\cos \theta, \sin \theta,-r^{\prime}\right) .
$$

The surface has constant mean curvature $H$ if

$$
2 H=-\frac{1}{r \sqrt{1+r^{\prime 2}}}+\frac{r^{\prime \prime}}{\left(1+r^{\prime 2}\right)^{3 / 2}} \text {. }
$$


Multiplying by $r r^{\prime}$ and integrating we see there exists $c \in \mathbb{R}$ such that

$$
H r^{2}=-\frac{r}{\sqrt{1+r^{\prime 2}}}+c
$$

Since $H r^{2}$ is positive, $c$ needs to be positive. Then there exist $h, \rho$ and a solution $r:[-h, h] \rightarrow[0, \rho]$ to (1) such that $r$ is even and the initial value $r(0)=t>0$ is the minimum of $r$. Moreover, $r(h)=\rho, r^{\prime}(h)=+\infty$, and $H \rho^{2}=c$. The associated surface is a nodoid.

For $u=0$, we have $H t^{2}+t=c$, so

$$
t=\frac{-1+\sqrt{1+4 H c}}{2 H} ;
$$

that is, $t$ an increasing function of $c$ with $t=0$ for $c=0$ and $\lim _{c \rightarrow+\infty} t=+\infty$. We will use $t$ as the parameter for the family of nodoids. We have

$$
\rho_{t}=\rho_{t}(H)=\sqrt{\frac{H t^{2}+t}{H}}, \quad h_{t}=h_{t}(H)=\int_{t}^{\rho} \frac{H\left(\rho^{2}-x^{2}\right)}{\sqrt{x^{2}-H^{2}\left(\rho^{2}-x^{2}\right)^{2}}} \mathrm{~d} x .
$$

To summarize:

Proposition 1. There exists a one-parameter family of nodoids $\left\{\mathcal{N}_{t}, t>0\right\}$ with constant mean curvature $H$ given by the rotation of a curve $\gamma_{t}$ around the $z$-axis, with the following properties:

(1) The curve $\gamma_{t}$ is a graph on $\left[h_{t}, h_{t}\right]$ of an even function.

(2) The curve $\gamma_{t}$ has horizontal tangents at $\pm h_{t}$. The surface $\mathcal{N}_{t}$ is included in the slab $\mathscr{Y}_{t}:|z| \leq h_{t}$ and is tangent to it.

(3) The mean curvature vector points out of the bounded domain determined by $\mathcal{N}_{t}$ in the slab $\mathscr{Y}_{t}$.

(4) The circle $C_{t}$ of $\mathcal{N}_{t}$ with smallest radius is given by $x^{2}+y^{2}=t^{2}, z=0$.

(5) The function $h_{t}$ is strictly increasing on $t$ and

$$
\lim _{t \rightarrow 0} h_{t}=0, \quad \lim _{t \rightarrow+\infty} h_{t}=\frac{1}{2 H} .
$$

(6) The function $\rho_{t}(H)$ is strictly increasing and

$$
\lim _{t \rightarrow 0} \rho_{t}(H)=0, \quad \lim _{t \rightarrow+\infty} \rho_{t}(H)=+\infty, \quad \lim _{t \rightarrow+\infty} \rho_{t}(H)-t=\frac{1}{2 H} .
$$

The two limits of $h_{t}$ and $\rho_{t}(H)$ as $t \rightarrow+\infty$ allow us to consider Collin's result as a limiting case of López's theorem. Indeed, when $R$ goes to $+\infty$, the uniform $R$-circle condition for $f$ becomes convexity, since the circle becomes a line. 


\section{The maximal and minimal solutions}

Solutions of the constant mean curvature Dirichlet problem (CMC) are bounded above by those of the corresponding zero mean curvature problem:

Lemma 2. Let $f: \mathbb{R} \rightarrow \mathbb{R}$ be a continuous function. On $\Omega=\mathbb{R} \times(-l, l)$, there exists a solution $w$ of the minimal surface equation (MSE) with $\left.w\right|_{\partial \Omega}=\varphi_{f}$. Moreover, $w \geq u$ for every solution $u$ of $(\mathrm{CMC})$ on $\Omega$ agreeing with $\varphi_{f}$ on the boundary.

Proof. By [Jenkins and Serrin 1966], if $n$ is a large enough integer, there exist solutions $w_{n}^{+}$and $w_{n}^{-}$of (MSE) on $(-n, n) \times(-l, l)$ with $w_{n}^{ \pm}=\varphi_{f}$ on $(-n, n) \times$ $\{-l, l\}$ and $w_{n}^{ \pm}= \pm \infty$ on $\{-n, n\} \times(-l, l)$. Fix such solutions for each $n$ large. By the maximum principle, for every $n$ and $m$, we have $w_{n}^{+} \geq w_{m}^{-}$, and $\left(w_{n}^{+}\right)$ is a decreasing sequence. Thus $\left(w_{n}^{+}\right)$converges to a solution $w$ of (MSE) on $\Omega$ agreeing with $\varphi_{f}$ on the boundary.

Now consider a solution $u$ of (CMC) on $\Omega$ with $\varphi_{f}$ as boundary value. By the maximum principle, $w_{n}^{+} \geq u$ for every $n$. Taking the limit, we see that $w \geq u$.

This gives an upper bound for $u$ without any hypothesis on the function $f$. To get a lower bound we do need such hypotheses.

The function $c$ defined on $\Omega=\mathbb{R} \times(-1 /(2 H), 1 /(2 H))$ by

$$
c(x, y)=-\frac{1}{\cos \theta} \sqrt{\frac{1}{4 H^{2}}-y^{2}}+\left(x-x_{0}\right) \tan \theta+z_{0}
$$

is a solution of (CMC): its graph is the half-cylinder with the two straight lines of equation $z=\left(x-x_{0}\right) \tan \theta+z_{0}$ over $\partial \Omega$ as boundary.

Lemma 3. Let $f: \mathbb{R} \rightarrow \mathbb{R}$ be a convex function and let $u$ be a solution of (CMC) on $\Omega=\mathbb{R} \times(-1 /(2 H), 1 /(2 H))$ agreeing with $\varphi_{f}$ on the boundary. Take $x_{0} \in \mathbb{R}$ and let $z=\left(x-x_{0}\right) \tan \theta_{0}+f\left(x_{0}\right)$ be a straight line lying below the graph of $f$ (such a line exists by convexity). Let $c$ denote the half-cylinder associated to this line. Then $u \geq c$ on $\Omega$.

Proof. Let $h$ be the function defined on $\Omega$ by $h(x, y)=\left(x-x_{0}\right) \tan \theta_{0}+f\left(x_{0}\right)$. We have $u \geq h$ on the boundary. If the function $f$ is affine, $f(x)=\left(x-x_{0}\right) \tan \theta_{0}+$ $f\left(x_{0}\right)$, Theorem 8 in [Mazet 2006a] states that $c$ is the only constant mean curvature extension for $\varphi_{f}$. Then $u=c$.

If $f$ is not affine, the set of $\theta$ such that there exists $x_{1} \in \mathbb{R}$ with $z=\left(x-x_{1}\right) \tan \theta+$ $f\left(x_{1}\right)$ lies below the graph of $f$ is an interval $I \subset \mathbb{R}$. We assume that $\theta_{0}$ is in the interior of this interval. For $\theta_{0}$ an end point of this interval, the property is proved by continuity.

Since $\theta_{0}$ is in the interior of $I$, there exist $x_{1}<x_{0}<x_{2}$ and $\theta_{1}<\theta_{0}<\theta_{2}$ such that $\left(x-x_{1}\right) \tan \theta_{1}+f\left(x_{1}\right) \leq f$ and $\left(x-x_{2}\right) \tan \theta_{2}+f\left(x_{2}\right) \leq f$. By Proposition 3 
in [Mazet 2006a], there exists $K \in \mathbb{R}_{+}$such that

$$
\begin{aligned}
& u(x, y) \geq\left(x-x_{1}\right) \tan \theta_{1}+f\left(x_{1}\right)-K, \\
& u(x, y) \geq\left(x-x_{2}\right) \tan \theta_{2}+f\left(x_{2}\right)-K .
\end{aligned}
$$

Since $\theta_{1}<\theta_{0}<\theta_{2}$, these two equations imply that $u(x, y) \geq h(x, y)$ if $|x|$ is big enough. We have $h \geq c$ on $\Omega$; then $u \geq c$ on $\partial \Omega$ and outside a compact of $\bar{\Omega}$. By the maximum principle, $u \geq c$ in $\Omega$.

In the case of the López solutions, we get the following lower bound.

Lemma 4. Let $f: \mathbb{R} \rightarrow \mathbb{R}$ a continuous function that satisfies a lower $\rho_{t}$-circle condition. Let $x$ be in $\mathbb{R}$ and let $\mathscr{C}$ be a circle of radius $\rho_{t}$ that established the uniform $\rho_{t}$-circle condition at the point $(x, f(x))$. Let $u$ be a solution of (CMC) on $\Omega=\mathbb{R} \times\left(-h_{t}, h_{t}\right)$ agreeing with $\varphi_{f}$ on the boundary. Then the graph of $u$ lies above the nodoid $\mathcal{N}_{t}$ having a horizontal axis and bounded by the two parallel circles $\mathscr{b}$ in the vertical plane $y=-h_{t}$ and $y=h_{t}$.

Proof. Let $e_{z}$ denote the vertical unit vector $(0,0,1)$. For $s$ in $\mathbb{R}$, we translate by $s e_{z}$ the nodoid $\mathcal{N}_{t}$ bounded by the two parallel circles $\mathscr{C}$. For $s$ negative enough, $\mathcal{N}_{t}+s e_{z}$ lies below the graph of $u$. Let $s$ grow until the first contact. The mean curvature of the graph is upward pointing and the mean curvature of $\mathcal{N}_{t}$ points outward. So by maximum principle, the first contact cannot be an interior point. Then, because of the hypothesis on $f$, the first contact is at $s=0$ and the lemma is proved.

The estimates in the preceding two lemmas have important consequences for uniqueness. To begin with, we derive from them a technical lemma.

Lemma 5. Let $f: \mathbb{R} \rightarrow \mathbb{R}$ be a continuous function such that either

(1) $\Omega=\mathbb{R} \times\left(-\frac{1}{2 H}, \frac{1}{2 H}\right)$ and $f$ is convex, or

(2) $\Omega=\mathbb{R} \times\left(-h_{t}, h_{t}\right)$ and $f$ satisfies a lower $\rho_{t}$-circle condition.

Let $\mathscr{D}$ denote the set of all solutions $u$ of $(\mathrm{CMC})$ on $\Omega$ agreeing with $\varphi_{f}$ on the boundary. For any $u_{1}, u_{2} \in \mathscr{D}$, there exist $v^{+}$and $v^{-}$in $\mathscr{D}$ such that

$$
v^{+} \geq \max \left(u_{1}, u_{2}\right), \quad v^{-} \leq \min \left(u_{1}, u_{2}\right) .
$$

Proof. For $n \in \mathbb{N}$, define

$$
\Omega_{n}=\left\{(x, y) \in \Omega \mid-n-\sqrt{1 /(2 H)^{2}-y^{2}} \leq x \leq n+\sqrt{1 /(2 H)^{2}-y^{2}}\right\} .
$$

The boundary of $\Omega$ is composed of two segments and two circle-arcs of curvature $2 H$. Following Perron's method (see [Courant and Hilbert 1962] or [Gilbarg and Trudinger 1983]), we build solutions $v_{n}^{+}$and $v_{n}^{-}$of (CMC) on $\Omega_{n}$, with $v_{n}^{+}=$ $\max \left(u_{1}, u_{2}\right)$ and $v_{n}^{-} \min \left(u_{1}, u_{2}\right)$ on the boundary. 
To build $v_{n}^{+}$, we consider subsolutions, of which $\max \left(u_{1}, u_{2}\right)$ is one. By the maximum principle, every subsolution is less than the solution $w$ of (MSE) given by Lemma 2 . We can then define $v_{n}^{+}$as the supremum over all subsolutions, and this function takes the right boundary values on the two segments because $\max \left(u_{1}, u_{2}\right)$ equals $w$ on it. For the two arcs of circle, we use the barrier functions built in [Serrin 1970].

Similarly, we define $v_{n}^{-}$as the infimum of all supersolutions, which exist since $\min \left(u_{1}, u_{2}\right)$ is one. Again by the maximum principle, every supersolution satisfies the lower bound in Lemma 3 or 4 . The half-circles and nodoids of these same lemmas are used as barrier functions and give us the boundary value of $v_{n}^{-}$on the two segments. For the two arcs of circle, we use Serrin's arguments.

On $\Omega_{n}$, we have $\max \left(u_{1}, u_{2}\right) \leq v_{n}^{+} \leq w$; thus a subsequence converges to $v^{+}$ on $\Omega$ and $v^{+} \in \mathscr{D}$. Clearly $\max \left(u_{1}, u_{2}\right) \leq v^{+}$. The sequence $v_{n}^{-}$is bounded above by $\min \left(u_{1}, u_{2}\right)$ and satisfies the lower bounds of Lemmas 3 or 4 . Therefore a subsequence converges to $v^{-}$a solution of (CMC) agreeing with $\varphi_{f}$ on the boundary. Moreover, $\min \left(u_{1}, u_{2}\right) \geq v^{-}$.

Proposition 6. Let $f: \mathbb{R} \rightarrow \mathbb{R}$ be a continuous function such that either

(1) $\Omega=\mathbb{R} \times\left(-\frac{1}{2 H}, \frac{1}{2 H}\right)$ and $f$ is convex, or

(2) $\Omega=\mathbb{R} \times\left(-h_{t}, h_{t}\right)$ and $f$ satisfies a lower $\rho_{t}$-circle condition.

There exist two solutions $u_{\max }$ and $u_{\min }$ of $(\mathrm{CMC})$ on $\Omega$ agreeing with $\varphi_{f}$ on the boundary and such that every solution $u$ of $(\mathrm{CMC})$ on $\Omega$ agreeing with $\varphi_{f}$ on the boundary satisfies

$$
u_{\min } \leq u \leq u_{\max } .
$$

Proof. Denote by $\mathscr{D}$ the set of all solutions $u$ of (CMC) on $\Omega$ agreeing with $\varphi_{f}$ on the boundary; by the work of Collin and López, $\mathscr{D}$ is nonempty. Define $u_{\max }$ and $u_{\min }$ at $p \in \Omega$ by

$$
u_{\max }(p)=\sup _{u \in \mathscr{D}} u(p), \quad u_{\min }(p)=\inf _{u \in \mathscr{D}} u(p) .
$$

By Lemma 2, $u_{\max }$ is well defined; Lemmas 3 and 4 ensure that $u_{\min }>-\infty$. As in the classical Perron process, it can be proved that $u_{\max }$ and $u_{\min }$ are solutions of (CMC) on $\Omega$ : the argument we need is that for every $u_{1}$ and $u_{2}$ in $\mathscr{D}$ there exist $u_{3} \in \mathscr{D}$ bounding $\max \left(u_{1}, u_{2}\right)$ from above and $u_{4} \in \mathscr{D}$ bounding $\min \left(u_{1}, u_{2}\right)$ from below. These are given by Lemma 5 .

Using the solution $w$ of (MSE) built in Lemma 2, the half-cylinders of Lemma 3 or the nodoids of Lemma 4 as barrier functions, we finally prove that $u_{\max }$ and $u_{\min }$ have $\varphi_{f}$ as boundary value. The construction also gives, for every $u \in \mathscr{D}$,

$$
u_{\min } \leq u \leq u_{\max } .
$$


An important fact is that, for every $(x, y) \in \Omega$, these solutions satisfy

$$
u_{\max }(x, y)=u_{\max }(x,-y), \quad u_{\min }(x, y)=u_{\min }(x,-y),
$$

because the functions $(x, y) \mapsto u_{\max }(x,-y)$ and $(x, y) \mapsto u_{\min }(x,-y)$ lie in $\mathscr{D}$.

Upper bounds. We now look for explicit upper bounds for solutions of (CMC).

Proposition 7. Let $f: \mathbb{R} \rightarrow \mathbb{R}$ be a continuous function and take $x_{0} \in \mathbb{R}$. Assume that $f$ is monotonic on $\left[x_{0},+\infty\right)$. Let $u$ be a solution of $(\mathrm{CMC})$ on $\Omega=\mathbb{R} \times(-a, a)$ agreeing with $\varphi_{f}$ on the boundary. Then, for $x \geq x_{0}+1 / H$, we have

$$
u(x, y) \leq f(x)+\frac{1}{2 H} .
$$

Proof. We only consider the case where $f$ is increasing on $\left[x_{0},+\infty\right)$. Take $a \geq x_{0}+$ $1 / H$ and denote by $C(s)$ the horizontal cylinder of axis $\{x=a-1 /(2 H)\} \cap\{z=s\}$ and radius $1 /(2 H)$. For $s$ large, $C(s)$ lies above the graph of $u$. Let $s$ decrease down to the value $s_{0}$ where the first contact happens. By the maximum principle, this first contact point is on the boundary at a point of first coordinate $a^{\prime} \in[a-1 /(2 H), a]$. We have $f\left(a^{\prime}\right) \geq s_{0}-1 /(2 H)$.

Since $C(s)$ lies above the graph of $u$ for every $s \geq s_{0}$, we have $u(a, y) \leq s$. Thus $u(a, y) \leq s_{0} \leq f\left(a^{\prime}\right)+1 /(2 H)$. Since $a^{\prime}<a$ and $f$ is increasing, we conclude that $u(a, y) \leq f(a)+1 /(2 H)$.

We say that a function $f: \mathbb{R} \rightarrow \mathbb{R}$ satisfies an upper $R$-circle condition at $a \in \mathbb{R}$ if $-f$ satisfies a lower $R$-circle condition there.

Remark. For large $s$, the disk with center $(a, s)$ and radius $R$ is entirely contained in $\left\{(x, y) \in \mathbb{R}^{2} \mid y \geq f(x)\right\}$. As $s$ decreases and first makes contact with the graph of $f$, we obtain an upper $R$-circle condition at the abscissa(s) of the contact point(s). As $a$ changes, we get all the abscissas where $f$ satisfies an upper $R$-circle condition. Thus for every $a \in \mathbb{R}$ there exists $a^{\prime} \in[a-R, a+R]$ where $f$ satisfies an upper $R$ circle condition.

Proposition 8. Let $f: \mathbb{R} \rightarrow \mathbb{R}$ be a continuous function. Let $u$ be a solution of $(\mathrm{CMC})$ on $\Omega=\mathbb{R} \times(-l, l)$ agreeing with $\varphi_{f}$ on the boundary. Assume the $f$ satisfies an upper $1 /(2 H)$-circle condition at $x_{0} \in \mathbb{R}$. Then $u\left(x_{0}, y\right) \leq f\left(x_{0}\right)$ for every $y \in[-l, l]$.

Proof. Let $\Gamma$ be a circle realizing the upper $1 /(2 H)$-circle condition at $x_{0}$. Denote by $C(s)$ the horizontal cylinder of axis $\{x=a\} \cap\{z=b+s\}$ and radius $1 /(2 H)$, where $(a, b)$ is the center of $\Gamma$. For big $s$ the cylinder $C(s)$ lies above the graph of $u$; as $s$ decreases, the first contact with the graph of $f$ happens for $s=0$, because of maximum principle, Then, on the segment $I_{x_{0}}=\left\{x_{0}\right\} \times[-l, l], u$ is bounded above by $f\left(x_{0}\right)$. 
Let $f: \mathbb{R} \rightarrow \mathbb{R}$ be a continuous function that satisfies a lower $R$-circle condition. Let $a \in \mathbb{R}$ denote a point where $f$ satisfies an upper $R^{\prime}$-circle condition. Since at $a$ there are circles both above and below the graph of $f$, the graph has a tangent there. Thus either $f^{\prime}(a)$ exists or $f^{\prime}(a)= \pm \infty$; either way, the derivative of $f$ at $a$ has a well defined sign. We have an analog of Rolle's Theorem:

Lemma 9. Let $f: \mathbb{R} \rightarrow \mathbb{R}$ be a continuous function that satisfies a lower $R$-circle condition. Let $a<b$ be two points where $f$ satisfies an upper $R^{\prime}$-circle condition. If $f^{\prime}(a)>0$ and $f^{\prime}(b)<0$, there exists $c \in[a, b]$ such that

(1) $f$ satisfies an upper $R^{\prime}$-circle condition at c, and

(2) $f^{\prime}(c)=0$.

Proof. Let $g$ denote the function defined by $g(x)=R^{\prime}-\sqrt{R^{\prime 2}-x^{2}}$ on $\left[-R^{\prime}, R^{\prime}\right]$; its graph is a half-circle of radius $R^{\prime}$. Since $f$ satisfies an upper $R^{\prime}$-circle condition at $a$ and $f^{\prime}(a)>0, f$ is upper bounded by $f(a)+g(x-a)$ on $\left[a-R^{\prime}, a\right]$. In the same way, $f$ is bounded above by $f(b)+g(x-b)$ on $\left[b, b+R^{\prime}\right]$. Let $c \in[a, b]$ denote a point where $f(c)=\max _{[a, b]} f$. Then $f(x)$ is bounded above by $m(x)$ on $\left[a-R^{\prime}, b+R^{\prime}\right]$, where $m(x)$ is defined by

$$
m(x)= \begin{cases}f(c)+g(x-a) & \text { for } x \in\left[a-R^{\prime}, a\right], \\ f(c) & \text { for } x \in[a, b], \\ f(c)+g(x-b) & \text { for } x \in\left[b, b+R^{\prime}\right] .\end{cases}
$$

This implies that $f$ satisfies an upper $R^{\prime}$-circle condition at $c$, so $f^{\prime}(c)=0$.

\section{The uniqueness of Collin and Wang's solutions}

Theorem 10. If $f: \mathbb{R} \rightarrow \mathbb{R}$ is a convex function, there is a unique solution of (CMC) on $\Omega=\mathbb{R} \times(-1 /(2 H), 1 /(2 H))$ agreeing with $\varphi_{f}$ on the boundary.

Proof. Existence is Collin's theorem (page 366); we prove uniqueness. By Proposition 6 , there are two solutions $u_{\min }$ and $u_{\max }$ of (CMC) on $\Omega$ agreeing with $\varphi_{f}$ on the boundary and such that, for every solution $u$ of the same Dirichlet problem, $u_{\min } \leq u \leq u_{\max }$. Thus our task is to show that $u_{\min }=u_{\max }$.

Suppose otherwise; then $u_{\max }-u_{\min }$ is unbounded on $\Omega$, by [Miklyukov 1979; Hwang 1988; Collin and Krust 1991]. By interchanging $x$ and $-x$ if needed, we can assume that

$$
\lim _{x \rightarrow+\infty} \max _{I_{x}}\left(u_{\max }-u_{\min }\right)=+\infty,
$$

where $I_{x}=\{x\} \times[-1 /(2 H), 1 /(2 H)]$.

Since $f$ is convex, $f$ has a left derivative $f_{l}^{\prime}$ and a right derivative $f_{r}^{\prime}$ at every point. These two functions increase and have the same limit at $+\infty$. If $\lim _{+\infty} f_{l}^{\prime}=$ 
$\lim _{+\infty} f_{r}^{\prime}<+\infty, f$ is lipschitz continuous on $\mathbb{R}_{+}$. Then (3) is in contradiction with [Mazet 2006a, Theorem 5]. Thus $f$ must satisfy

$$
\lim _{+\infty} f_{l}^{\prime}=\lim _{+\infty} f_{r}^{\prime}=+\infty \text {. }
$$

Asymptotic behavior of $\boldsymbol{u}_{\text {min. }}$. To proceed we must recall from [Tam 1987a; 1987b; Mazet 2006b] the notion of an arc of divergence. Let $\left(v_{n}\right)$ be a sequence of solutions of (CMC) and let $N_{n}$ denote the upward pointing normal to the graph of $v_{n}$. Assume that $N_{n}(P)$ tends to a horizontal unit vector $(v, 0)$, with $v \in \mathbb{S}^{1}$. Let $C$ denote the arc of circle in the $x y$-plane with radius $1 /(2 H)$ such that $P$ lies in $C$ and $2 H v$ is the curvature vector of $C$ at $P$. We call $C$ an arc of divergence of the sequence $\left(v_{n}\right)$. We extend $v$ to $C$ by letting $2 H v(Q)$ be the curvature vector of $C$ at $Q \in C$. Then $N_{n}(Q)$ converges to $(v(Q), 0)$ for every $Q \in C$.

For $u$ a function on a domain of $\mathbb{R}^{2}$, define the differential 1 -form $\omega_{u}$ by

$$
\omega_{u}=\frac{u_{x}}{\sqrt{1+|\nabla u|^{2}}} \mathrm{~d} y-\frac{u_{y}}{\sqrt{1+|\nabla u|^{2}}} \mathrm{~d} x,
$$

where $u_{x}$ and $u_{y}$ are the partial derivatives of $u$. When $u$ is a solution of (CMC), $\omega_{u}$ satisfies $\mathrm{d} \omega_{u}=2 H \mathrm{~d} x \wedge \mathrm{d} y$; see [Spruck 1972/73]. It follows from the previous paragraph that, for every subarc $C^{\prime}$ of the arc of divergence $C$, we have

$$
\lim _{n \rightarrow+\infty} \int_{C^{\prime}} \omega_{u}=\ell\left(C^{\prime}\right)
$$

where $\ell\left(C^{\prime}\right)$ the length of $C^{\prime}$. We orient $C^{\prime}$ so that $v$ points toward the left along $C^{\prime}$.

For $a \in \mathbb{R}$, denote by $C^{+}(a)$ the arc of circle

$$
\{x \geq a\} \cap\left\{(x-a)^{2}+y^{2}=1 /\left(4 H^{2}\right)\right\} .
$$

Its endpoints are $(a, \pm 1 /(2 H))$ and it contains the point $(a+1 /(2 H), 0)$.

Lemma 11. There exists an increasing real sequence $\left(x_{n}\right)$ such that $\lim x_{n}=+\infty$ and $C^{+}(0)$ is an arc of divergence of the sequence $\left(u_{n}\right)$ of solutions of (CMC) on $\Omega$, where $u_{n}$ is defined by $u_{n}(x, y)=u_{\min }\left(x+x_{n}, y\right)$.

Proof. Let $v_{n}$ be defined on $\Omega$ by $v_{n}(x, y)=u_{\min }(x+n, y)$. The boundary value of $v_{n}$ is $\varphi_{f_{n}}$ with $f_{n}(x)=f(x+n)$. Because of $(4), f_{n}$ is increasing on $[-1 / H,+\infty)$ for $n$ large. Hence, by Proposition 7, $v_{n}(0,0) \leq f_{n}(0)+1 /(2 H)$. Now let $\theta_{n} \in$ $[0, \pi / 2)$ be such that $f_{n_{l}}^{\prime}(0) \leq \tan \theta_{n} \leq f_{n_{r}}^{\prime}(0)$. By Lemma 3 we have

$$
v_{n}\left(\frac{1}{H}, 0\right) \geq-\frac{1}{\cos \theta_{n}} \sqrt{\frac{1}{4 H^{2}}}+\frac{1}{H} \tan \theta_{n}+f_{n}(0) .
$$

Again because of (4), $\theta_{n}$ converges to $\rightarrow \pi / 2$. Hence

$$
v_{n}\left(\frac{1}{H}, 0\right)-v_{n}(0,0) \geq \frac{1}{H \cos \theta_{n}}\left(\sin \theta_{n}-\frac{1}{2}\right)-\frac{1}{2 H} \underset{n \rightarrow+\infty}{\longrightarrow}+\infty .
$$


Thus the sequence of derivatives $\partial v_{n} / \partial x$ cannot stay bounded above on the segment $[0,1 / H] \times\{0\}$; that is, there exists a sequence $\left(a_{n}\right)$ in $[0,1 / H]$ such that

$$
\lim \frac{\partial v_{n}}{\partial x}\left(a_{n}, 0\right)=+\infty
$$

If we set $x_{n}=n+a_{n}-1 /(2 H),(5)$ becomes

$$
\lim \frac{\partial u_{n}}{\partial x}\left(\frac{1}{2 H}, 0\right)=+\infty .
$$

Since $\partial u_{n} / \partial y(1 /(2 H), 0)=0$ by (2), the limit normal to the sequence of graphs over $(1 /(2 H), 0)$ is $(-1,0,0)$. Therefore $C^{+}(0)$ is a line of divergence for $\left(u_{n}\right)$. By passing to a subsequence we can assume that $\left(x_{n}\right)$ is increasing, and clearly $\lim x_{n}=+\infty$. This proves Lemma 11 .

Conclusion of proof. Let $\left(x_{n}\right)$ be as in Lemma 11. Recalling the limit (3) and the surrounding notation, define

$$
c:=\max _{I_{0}}\left(u_{\max }-u_{\min }\right) .
$$

The set $\left\{(x, y): u_{\max }(x, y) \geq u_{\min }(x, y)+2 c\right\}$ has a connected component $W$ contained in $\mathbb{R}_{+} \times[-1 /(2 H), 1 /(2 H)]$. This component is unbounded. Now define

$$
W_{n}=W \cap\left\{(x, y) \in \Omega \mid x \leq x_{n}+\sqrt{1 /\left(4 H^{2}\right)-y^{2}}\right\} .
$$

The boundary of $W_{n}$ is the union of $\partial W \cap W_{n}$ and $\Gamma_{n}$, the latter being the part contained in the semicircle $C^{+}\left(x_{n}\right)$ :

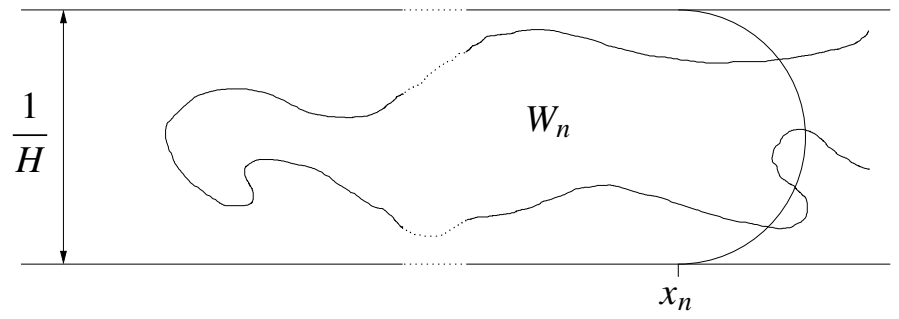

Set $\widetilde{\omega}=\omega_{u_{\max }}-\omega_{u_{\min }}$. Then

$$
0=\int_{\partial W_{n}} \widetilde{\omega}=\int_{\partial W \cap W_{n}} \widetilde{\omega}+\int_{\Gamma_{n}} \widetilde{\omega} .
$$

By Lemma 2 in [Collin and Krust 1991], the integral on $\partial W \cap W_{n}$ is negative; and it decreases as $n$ increases, since $\left(x_{n}\right)$ is increasing. Moreover,

$$
0<-\int_{\partial W \cap W_{n}} \tilde{\omega}=\int_{\Gamma_{n}} \widetilde{\omega} \leq 2 \ell\left(\Gamma_{n}\right)
$$


where $\ell\left(\Gamma_{n}\right)$ is the length of $\Gamma_{n}$. Thus $\ell\left(\Gamma_{n}\right)$ is uniformly bounded away from 0 . Because of Lemma 11 and since $\Gamma_{n} \subset C^{+}\left(x_{n}\right)$, there exists a sequence $\left(\alpha_{n}\right)$ in $[0,1]$ such that $\lim \alpha_{n}=1$ and

$$
\int_{\Gamma_{n}} \omega_{u_{\min }} \geq \alpha_{n} \ell\left(\Gamma_{n}\right)
$$

Finally, for $n \geq n_{0}>0$, we have

$$
\begin{aligned}
-\int_{\partial W \cap W_{n_{0}}} \tilde{\omega} & \leq-\int_{\partial W \cap W_{n}} \tilde{\omega}=\int_{\Gamma_{n}} \omega_{u_{\max }}-\int_{\Gamma_{n}} \omega_{u_{\min }} \\
& \leq \ell\left(\Gamma_{n}\right)-\alpha_{n} \ell\left(\Gamma_{n}\right) \leq\left(1-\alpha_{n}\right) \ell\left(\Gamma_{n}\right) \underset{n \rightarrow+\infty}{\longrightarrow} 0 .
\end{aligned}
$$

But as we have seen the leftmost expression is strictly positive. This contradiction proves Theorem 10.

\section{The uniqueness of López's solutions}

Theorem 12. If $f: \mathbb{R} \rightarrow \mathbb{R}$ is a continuous function that satisfies a lower $\rho_{t}$-circle condition, there is a unique solution of $(\mathrm{CMC})$ on $\Omega=\mathbb{R} \times\left(-h_{t}, h_{t}\right)$ agreeing with $\varphi_{f}$ on the boundary.

Proof. Take $u_{\min }, u_{\max }, c, I_{x}$, and $W$ as in the proof of Theorem 10, but with $1 /(2 H)$ replaced by $h_{t}$ in the definition of $I_{x}$, and $W$. The limit (3) holds.

Lemma 13. There exists $x_{0} \in \mathbb{R}_{+}$such that $f$ is monotonic on $\left[x_{0},+\infty\right)$.

Proof. Consider the set $\mathscr{S}$ of points where $f$ satisfies an upper $1 /(2 H)$-circle condition. By the remark on page $372, \mathscr{S}$ is nonempty and unbounded, and at each point of $\mathscr{S}$ the (possibly infinite) derivative of $f$ has a well defined sign. We claim that there exists $x_{1} \in \mathbb{R}^{+}$such that $f^{\prime}(x)$ has constant sign for every $x \in \mathscr{Y} \cap\left[x_{1},+\infty\right)$. If not, take an increasing sequence of points in $\mathscr{S}$ tending to $+\infty$ and such that $f^{\prime}$ is alternately positive and negative at these points. Then Lemma 9 yields an increasing sequence $\left(c_{n}\right)$ in $\mathscr{S}$ tending to $+\infty$ and such that $f^{\prime}\left(c_{n}\right)=0$ for all $n$. Let $u$ be a solution of (CMC) on $\Omega$ agreeing with $\varphi_{f}$ on the boundary. By Proposition $8, \max _{I_{c_{n}}} u \leq f\left(c_{n}\right)$. Since $f^{\prime}\left(c_{n}\right)=0$, Lemma 4 implies that $\min _{I_{c_{n}}} u \geq f\left(c_{n}\right)-\left(\rho_{t}-t\right)$. Hence $\max _{I_{c_{n}}}\left(u_{\max }-u_{\min }\right) \leq \rho_{t}-t$, in contradiction with (3) since $\lim c_{n}=+\infty$. This proves the claim. We assume that $f^{\prime}(x)>0$ for $x$ large, the case $f^{\prime}(x)<0$ being handled similarly.

Now suppose the assertion of the lemma fails, so there is an increasing sequence $\left(a_{n}\right)$ in $\left[x_{1},+\infty\right)$ such that $\lim a_{n}=+\infty$ and $f\left(a_{n}\right)$ is a local maximum of $f$ for each $n$. Since $f$ satisfies a lower $\rho_{t}$-circle condition, $f$ is differentiable at every $a_{n}$. Let $\Gamma(s)$ be the circle of center $\left(a_{n}-1 /(2 H), s\right)$ and radius $1 /(2 H)$. For $s$ large, $\Gamma(s)$ lies above the graph of $f$; when $s$ decreases down to the first contact 
of $\Gamma(s)$ with the graph, we get a point $x$ where $f$ satisfies an upper $1 /(2 H)$-circle condition. By assumption $f^{\prime}(x)>0$, so $x \in\left[a_{n}-1 /(2 H), a_{n}\right]$. Let $b_{n} \in\left[x, a_{n}\right]$ be a point maximizing $f$ in $\left[x, a_{n}\right]$. Since $f^{\prime}(x)>0$, we have $b_{n} \in\left(x, a_{n}\right]$, hence $f^{\prime}\left(b_{n}\right)=0$. Using horizontal cylinders with $\Gamma(s)$ as vertical section, we prove that $\max _{I_{b_{n}}} u \leq f(x)+1 /(2 H) \leq f\left(b_{n}\right)+1 /(2 H)$, where $u$ a solution of (CMC) on $\Omega$ agreeing with $\varphi_{f}$ on the boundary. Also, since $f^{\prime}\left(b_{n}\right)=0$, we have $\min _{I_{b_{n}}} u \geq$ $f\left(b_{n}\right)-\left(\rho_{t}-t\right)$. Thus $\max _{I_{b_{n}}}\left(u_{\max }-u_{\min }\right) \leq 1 /(2 H)+\left(\rho_{t}-t\right)$. But $\lim b_{n}=+\infty$, so this last inequality contradicts $(3)$, proving the lemma.

We now assume that $f$ is increasing on $\left[x_{0},+\infty\right)$; the case of $f$ decreasing is handled similarly. By Theorem 5 in [Mazet 2006a], we know that $f(x+4 / H)-$ $f(x)$ cannot stay bounded as $x$ goes to $+\infty$. We even know that

$$
\lim _{x \rightarrow+\infty} f(x+4 / H)-f(x)=+\infty .
$$

This identity will play the same role as (4) in the proof of Theorem 10.

Asymptotic behavior of $\boldsymbol{u}_{\text {min }}$. For $a \in \mathbb{R}$, denote by $C^{+}(a)$ the arc of circle

$$
\{x \geq a\} \cap\left\{\left(x-\left(a-\sqrt{\left(1 / 4 H^{2}\right)-h_{t}^{2}}\right)\right)^{2}+y^{2}=\left(1 / 4 H^{2}\right)\right\} .
$$

Its endpoints are $\left(a, \pm h_{t}\right)$ and it contains the point $(a+K, 0)$, where

$$
K=\frac{1}{2 H}-\sqrt{\frac{1}{4 H^{2}}-h_{t}^{2}} .
$$

Next we claim that Lemma 11 holds verbatim in this setting; that is, there exists an increasing, diverging real sequence $\left(x_{n}\right)$ such that $C^{+}(0)$ is an arc of divergence of the sequence $\left(u_{n}\right)$ of translates of $u_{\min }$ by $\left(-x_{n}, y\right)$. To see this, let $v_{n}$ be the translate defined on $\Omega$ by $v_{n}(x, y)=u_{\min }(x+n, y)$; its restriction to the boundary is $\varphi_{f_{n}}$, with $f_{n}(x)=f(x+n)$. For $n$ large enough, $f_{n}$ is increasing on $[1 / H,+\infty)$;

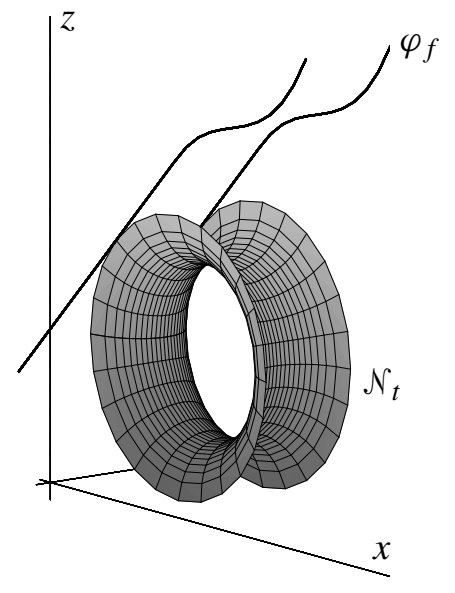


using Proposition 7, we get $v_{n}(0,0) \leq f_{n}(0)+1 /(2 H)$. Now apply Lemma 4 at $4 / H$ to see that the graph of $v_{n}$ lies above a nodoid $\mathcal{N}_{t}$ with horizontal axis in the vertical plane $x=4 / H+A\left(0 \leq A \leq \rho_{t}\right.$ since $f$ is increasing $)$. Since $\mathcal{N}_{t}$ lies below the graph, we have $v_{n}(4 / H+A, 0) \geq f_{n}(4 / H)-\left(\rho_{t}-t\right)$ (see figure on preceding page). Now translate $\mathcal{N}_{t}$ by the horizontal vector $e_{x}=(1,0,0)$; since $f_{n}$ is increasing, the nodoid $\mathcal{N}_{t}+s e_{x}$ does not cross the boundary, and so stays below the graph. Setting $s=\rho_{t}-A$ we then get

$$
v_{n}\left(\frac{4}{H}+\rho_{t}, 0\right) \geq f_{n}\left(\frac{4}{H}\right)-\left(\rho_{t}-t\right),
$$

which leads to

$$
v_{n}\left(\frac{4}{H}+\rho_{t}, 0\right)-v_{n}(0,0) \geq f_{n}\left(\frac{4}{H}\right)-f_{n}(0)-\frac{1}{2 H}-\rho_{t}+t .
$$

By (6) we have $\lim v_{n}\left(4 / H+\rho_{t}, 0\right)-v_{n}(0,0)=+\infty$. Hence the sequence of derivatives $\partial v_{n} / \partial x$ cannot stay bounded above on $\left[0,4 / H+\rho_{t}\right] \times\{0\}$; that is, there exists a sequence $\left(a_{n}\right)$ in $\left[0,4 / H+\rho_{t}\right]$ such that

$$
\lim \frac{\partial v_{n}}{\partial x}\left(a_{n}, 0\right)=+\infty
$$

If we set $x_{n}=n+a_{n}-K$, (7) becomes

$$
\lim \frac{\partial u_{n}}{\partial x}(K, 0)=+\infty
$$

Since $\partial u_{n} / \partial y(K, 0)=0$ by (2), the limiting normal to the sequence of graphs over $(K, 0)$ is $(-1,0,0)$. Therefore $C^{+}(0)$ is a line of divergence for $\left(u_{n}\right)$, and the claim is proved (see end of proof of Lemma 11).

To conclude the proof of our theorem (when $f$ is increasing beyond $x_{0}$ ) we simply repeat the reasoning in the last portion of the proof of Theorem 10, with the only difference that $W_{n}$ is defined as

$$
W_{n}=W \cap\left\{(x, y) \in \Omega \mid x \leq x_{n}+\sqrt{1 /\left(4 H^{2}\right)-y^{2}}-\sqrt{1 /\left(4 H^{2}\right)-h_{t}^{2}}\right\} .
$$

(To deal with the case where $f$ is decreasing we replace $u_{\min }$ by $u_{\max }$ and replace $C^{+}(a)$ by $C^{-}(a)$, defined by

$$
\left.C^{-}(a)=\{x \leq a\} \cap\left\{\left(x-\left(a+\sqrt{\left(1 / 4 H^{2}\right)-h_{t}^{2}}\right)\right)^{2}+y^{2}=\left(1 / 4 H^{2}\right)\right\} .\right)
$$

\section{References}

[Collin 1990] P. Collin, "Deux exemples de graphes de courbure moyenne constante sur une bande de $\mathbf{R}^{2}$, C. R. Acad. Sci. Paris Sér. I Math. 311:9 (1990), 539-542. MR 92i:58041 Zbl 0716.53016

[Collin and Krust 1991] P. Collin and R. Krust, "Le problème de Dirichlet pour l'équation des surfaces minimales sur des domaines non bornés”, Bull. Soc. Math. France 119:4 (1991), 443-462. MR 92m:53007 Zbl 0754.53013 
[Courant and Hilbert 1962] R. Courant and D. Hilbert, Methods of mathematical physics, II: Partial differential equations, Wiley, New York, 1962. Reprinted 1989. MR 90k:35001 Zbl 0099.29504

[Delaunay 1841] C. Delaunay, "Sur la surface de révolution dont la courbure moyenne est constante", J. Math. Pure Appl. 6 (1841), 309-320.

[Eells 1987] J. Eells, “The surfaces of Delaunay”, Mathematical Intelligencer 9:1 (1987), 53-57. MR 88h:53011 Zbl 0605.53002

[Finn 1965] R. Finn, "Remarks relevant to minimal surfaces, and to surfaces of prescribed mean curvature”, J. Analyse Math. 14 (1965), 139-160. MR 32 \#6337 Zbl 0163.34604

[Gilbarg and Trudinger 1983] D. Gilbarg and N. S. Trudinger, Elliptic partial differential equations of second order, 2nd ed., Grundlehren der Math. Wissenschaften 224, 1983. Corrected reprint, 1998. MR 2001k:35004 Zbl 0562.35001

[Huang 1995] W. H. Huang, "Boundedness of surfaces of constant sign mean curvature and the Phragmén-Lindelöf problem”, pp. 116-123 in Advances in geometric analysis and continuum mechanics (Stanford, 1993), edited by P. Concus and K. Lancaster, Int. Press, Cambridge, MA, 1995. MR 96g:53012 Zbl 0861.53010

[Hwang 1988] J.-F. Hwang, "Comparison principles and Liouville theorems for prescribed mean curvature equations in unbounded domains", Ann. Scuola Norm. Sup. Pisa Cl. Sci. (4) 15:3 (1988), 341-355. MR 90m:35019 Zbl 0705.49022

[Hwang 1995] J. F. Hwang, "On the uniqueness of capillary surfaces over an infinite strip", Pacific J. Math. 169:1 (1995), 95-105. MR 96f:58037 Zbl 0834.35050

[Jenkins and Serrin 1966] H. Jenkins and J. Serrin, "Variational problems of minimal surface type, II: Boundary value problems for the minimal surface equation", Arch. Rational Mech. Anal. 21 (1966), 321-342. MR 32 \#8221 Zbl 0171.08301

[López 2001] R. López, "Constant mean curvature graphs on unbounded convex domains”, J. Differential Equations 171:1 (2001), 54-62. MR 2002c:53011 Zbl 0987.35059

[López 2002] R. López, "Constant mean curvature graphs in a strip of $\mathbb{R}^{2}$ ”, Pacific J. Math. 206:2 (2002), 359-373. MR 2003j:53010 Zbl 01868190

[Mazet 2006a] L. Mazet, "A height estimate for constant mean curvature graphs and uniqueness results”, Manuscripta Math. 119:2 (2006), 255-268. MR 2215971 Zbl 1093.53014

[Mazet 2006b] L. Mazet, "Lignes de divergence pour les graphes à courbure moyenne constante", Ann. Inst. H. Poincaré Anal. Non Linéaire (2006).

[Miklyukov 1979] V. M. Miklyukov, “On a new approach to Bernstein's theorem and related questions for equations of minimal surface type", Mat. Sb. (N.S.) 108:2 (1979), 268-290. In Russian; translated in Math. USSR Sb. 36 (1980), 251-271. MR 80e:53005

[Nitsche 1965] J. C. C. Nitsche, "On new results in the theory of minimal surfaces", Bull. Amer. Math. Soc. 71 (1965), 195-270. MR 30 \#4200 Zbl 0135.21701

[Serrin 1969] J. Serrin, "On surfaces of constant mean curvature which span a given space curve.", Math. Z. 112 (1969), 77-88. MR 40 \#3447 Zbl 0182.24001

[Serrin 1970] J. Serrin, "The Dirichlet problem for surfaces of constant mean curvature", Proc. London Math. Soc. (3) 21 (1970), 361-384. MR 43 \#1093 Zbl 0199.16604

[Spruck 1972/73] J. Spruck, "Infinite boundary value problems for surfaces of constant mean curvature”, Arch. Rational Mech. Anal. 49 (1972/73), 1-31. MR 48 \#12329 Zbl 0263.53008

[Tam 1987a] L.-F. Tam, "On the uniqueness of capillary surfaces without gravity over an infinite strip”, Indiana Univ. Math. J. 36:1 (1987), 79-89. MR 88f:53011 Zbl 0678.49037 
[Tam 1987b] L.-F. Tam, "On the uniqueness of capillary surfaces", pp. 99-108 in Variational methods for free surface interfaces (Menlo Park, CA, 1985), edited by P. Concus and R. Finn, Springer, New York, 1987. MR 88c:53016 Zbl 0711.49060

[Wang 1990] A. N. Wang, "Constant mean curvature surfaces on a strip", Pacific J. Math. 145:2 (1990), 395-396. MR 91i:53016 Zbl 0724.49026

Received September 22, 2005.

\section{LAURENT MAZET}

UNIVERSité PAUl SABATIER, MIG

LABORATOIRE ÉMILE PICARD, UMR 5580

31062 TOULOUSE CEDEX 9

FRANCE

laurent.mazet@1mpt.univ-tours.fr

http://www.lmpt.univ-tours.fr/ mazet/ 